\section{Chronic longitudinal NSAID-related ulcer of the colon ("colon single-stripe sign") in Munchhausen syndrome}

A 62-year-old woman presented with chronic gastrointestinal bleeding. Colonoscopy showed a longitudinal ulcer extending from the ascending to the transverse colon ( $\bullet$ Fig. 1 a). Biopsies taken from the ulcer ruled out malignancy and inflammatory bowel disease. Despite extensive diagnostic workup, including angiography and CT scan, the cause of the ulcer remained unclear. The patient denied taking nonsteroidal anti-inflammatory drugs (NSAIDs). The patient received empiric treatment with antibiotics, steroids, and mesalamine (5-aminosalicylic acid, 5-ASA), but the ulcer persisted for 6 months on repeated endoscopy. During this period the patient required the transfusion of 17 units of red blood cells. Repeated gastrointestinal hemorrhage finally prompted right hemicolectomy.

The resection specimen showed a $23-\mathrm{cm}-$ long antimesenteric ulcer of the ascending and transverse colon with a maximum width of $3 \mathrm{~cm}$ ( Fig. 1 b). Histological analysis disclosed chronic ulceration with reactive epithelial changes (৫ Fig. 1c). Three months later the patient again presented with lower gastrointestinal bleeding. Colonoscopy showed a new longitudinal ulcer in the descending colon and additional smaller ulcers in the ileum ( Fig. 1d). Despite repeated denial regarding NSAID intake, diclofenac and its metabolites were detected in the patient's urine by high-performance liquid chromatography. In the face of these results, the patient finally admitted to chronic NSAID consumption.

NSAID-induced ulcers in the colon are usually reported as ring-like ulcers with the formation of diaphragms, or as wellcircumscribed single or multiple flat ulcers, especially in the right colon and around the ileocecal valve [1]. The unusual longitudinal appearance of the presented ulcer has previously been referred to as "colon single-stripe sign" and is thought to be related to ischemia [2]. The occurrence of the colon single-stripe sign in a patient with chronic NSAID intake and the antimesenteric location of the mucosal defect support the concept that NSAID-related colonic ulcers may be caused by local ischemic injury due to liberation of vasoconstrictive metabolites and/or vascular spasms [3].

Endoscopy_UCTN_Code_CCL_1AD_2AD
C. Hogenauer ${ }^{1}$, A. Eherer ${ }^{1}$, J. Pfeifer ${ }^{2}$, C. Langner ${ }^{3}$

Department of Internal Medicine, Division of Gastroenterology and Hepatology, Medical University, Graz, Austria

Department of Surgery, Medical University, Graz, Austria

Institute of Pathology, Medical University, Graz, Austria

\section{References}

1 Byrne MF, McGuinness J, Smyth CM et al. Nonsteroidal anti-inflammatory drug-induced diaphragms and ulceration in the colon. Eur J Gastroenterol Hepatol 2002; 14: $1265-1269$

2 Zuckerman GR, Prakash C, Merriman RB et al. The colon single-stripe sign and its relationship to ischemic colitis. Am J Gastroenterol 2003; 98: 2018-2022

3 Stolte $M$, Karimi $D$, Vieth $M$ et al. Strictures, diaphragms, erosions or ulcerations of ischemic type in the colon should always prompt consideration of nonsteroidal antiinflammatory drug-induced lesions. World J Gastroenterol 2005; 11: 5828 - 5833

\section{Bibliography}

DOI $10.1055 / \mathrm{s}-2007-995800$

Endoscopy 2008; 40: E233

(c) Georg Thieme Verlag KG Stuttgart · New York . ISSN 0013-726X

\section{Corresponding author}

\section{Langner, MD}

Institute of Pathology

Medical University of Graz

Auenbruggerplatz 25

8036 Graz

Austria
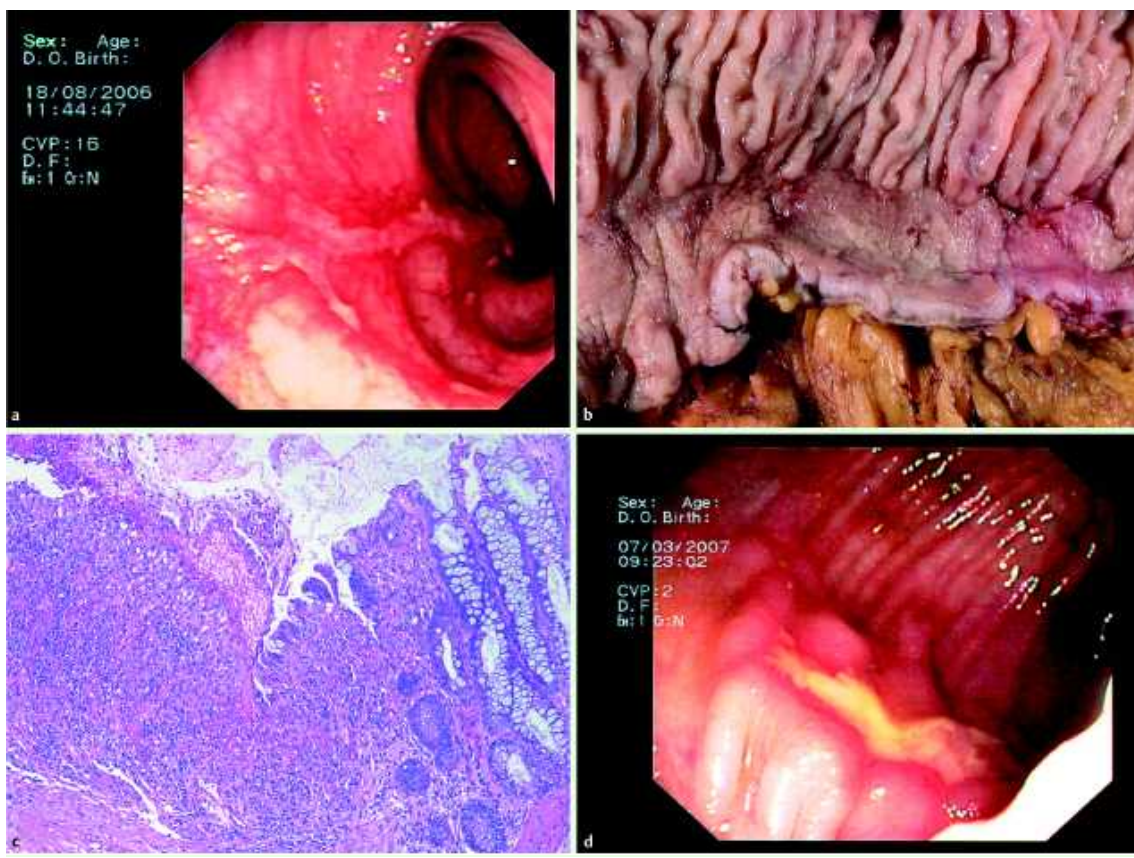

Fig. 1 At colonoscopy the patient is found to have a longitudinal ulcer extending from the ascending to the transverse colon (a). The resection specimen shows a 23-cm-long antimesenteric ulcer with a maximum width of $3 \mathrm{~cm}$ (b). Histological section shows chronic ulceration with mild reactive epithelial changes (c). Three months later a new longitudinal ulcer is detected in the descending colon together with additional, smaller ulcers in the ileum (d). 